\title{
Management of the aortic arch and descending thoracic aorta for acute type A dissection: Moving away from the "less is more" paradigm
}

\author{
Prashanth Vallabhajosyula, MD, MS, and Wilson Y. Szeto, MD
}

See related article on pages 101-5.

The treatment strategy for the aortic arch in the setting of acute type A dissection is evolving as improved surgical techniques, technologic advances with endovascular stent grafts, and refined circulatory management strategies have enabled cardiac surgeons to safely perform more extensive interventions on the aortic arch and descending thoracic aorta (DTA). This has led to progression from a clamped distal aortic anastomosis to an open transverse hemiarch replacement to total arch replacement, and now to a total arch replacement with concomitant stent grafting of the DTA (frozen elephant trunk [FET] procedure) as the empiric treatment strategy. Because of the potential technical complexities associated with a zone III (distal to the left subclavian artery [LSCA]) anastomosis for FET, Zhu and colleagues ${ }^{1}$ report in this issue of the Journal their outcomes with FET with zone II (distal to the left common carotid artery [LCCA]) anastomosis and end-to-side reimplantation of the LSCA to the LCCA. Their excellent postoperative outcomes should be commended, and even though the sample size is small, their study shows that at centers of excellence alternative surgical techniques for FET reconstruction can be safely used. The authors of this study report that "moving" the distal anastomosis from zone III to zone II makes the operation technically easier by "simplifying hemostasis and anastomosis."

There is no doubt that the introduction of advanced technology such as the FET has improved our ability to manage complex arch pathologies. But does this mean we should be doing the FET operation on every patient with type A dissection? If there is no primary intimal tear in the aortic arch or the proximal DTA, then should we empirically dissect the arch vessels and perform total arch

From the Division of Cardiovascular Surgery, University of Pennsylvania Medical Center, Penn Presbyterian Medical Center, Philadelphia, Pa.

Disclosures: Authors have nothing to disclose with regard to commercial support.

Received for publication March 30, 2015; accepted for publication April 1, 2015; available ahead of print May 13, 2015.

Address for reprints: Wilson Y. Szeto, MD, Division of Cardiovascular Surgery, University of Pennsylvania Medical Center, Penn Presbyterian Medical Center, 51 N 39th St, Heart and Vascular Pavilion 2A, Philadelphia, PA 19104 (E-mail: wilson.szeto@uphs.upenn.edu).

J Thorac Cardiovasc Surg 2015;150:106-7

$0022-5223 / \$ 36.00$

Copyright (c) 2015 by The American Association for Thoracic Surgery

http://dx.doi.org/10.1016/j.jtcvs.2015.04.003

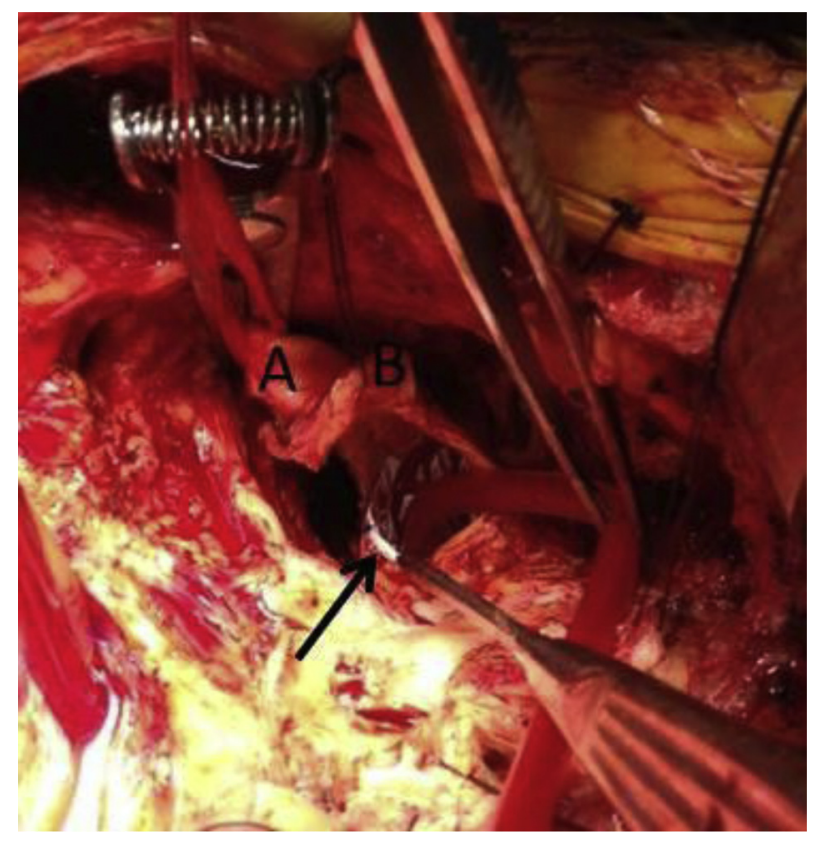

FIGURE 1. Antegrade stent grafting of the descending thoracic aorta during circulatory arrest is shown for a patient with type A aortic dissection.

replacement in an emergency situation? In the emergency situation, when the primary tear is not in the aortic arch or can be resected with transverse hemiarch, should we be dissecting the LSCA extensively by cutting into the "sternocleidomastoid muscle and the cervical muscle groups" in preparation for LSCA to LCCA reimplantation? Although the authors of this study should be congratulated on their excellent outcomes, it is not inconceivable that the use of the described technique for all cases of type A dissection could result in higher rates of injury to the recurrent laryngeal nerve, phrenic nerve, and thoracic duct, and it might also increase swallow dysfunction postoperatively. Some surgeons may argue that the level of mobilization required for LSCA reimplantation to the LCCA may negate the advantage of a zone II over zone III anastomosis. It may make the already complex care of patients with type A dissection even more challenging. In the study by Zhu and colleagues, ${ }^{1}$ zone II FET with LSCA reimplantation was reported in 20 patients, among whom the primary tear occurred in the ascending aorta in 8 patients $(40 \%)$ and the transverse arch in 2 patients $(10 \%)$. In addition, in the studied cohort, "surgery was performed within 2 weeks after the onset of pain." It is 
therefore unclear how applicable this strategy is in an emergency situation, as opposed to the subacute situation.

The FET operation is a more complex surgical endeavor than a transverse hemiarch reconstruction. In the acute dissection setting, total arch replacement with elephant trunk has been associated with increased morbidity and mortality. ${ }^{2,3}$ So if a total arch replacement is not required, then why do it? The major advantage of total arch replacement, especially FET, is distal aortic remodeling and reduction of distal reintervention rate. Unlike centers of excellence, where vast resources with concentrated technical and medical expertise exist, most operations for type A dissection occur in smaller, community hospital settings. This raises the issue of wide clinical application of the FET technique for acute type A dissection. So the more important decision is not zone II versus zone III FET, but FET versus transverse hemiarch reconstruction for acute type A dissection. To address this, a more comprehensive and meticulous approach is warranted on the basis of such parameters as patient clinical picture, acuity, malperfusion, arch and DTA anatomy, and primary tear site location. We believe that the current literature does not support empirical total arch replacement relative to transverse hemiarch reconstruction. A more sophisticated set of selection criteria needs to be established in combination with available surgical expertise in making the decision to perform the FET operation. In treating patients in whom the primary arch tear cannot be resected or occurs in the proximal DTA, however, the FET operation will have an important role. In this subpopulation of patients with type A dissection, the zone II FET with LSCA implantation may be an important alternative strategy to zone III FET.

Recently a few groups, including our institution, have advocated for transverse hemiarch reconstruction with DTA stent grafting as a distal reconstructive strategy (Figure 1). ${ }^{4,5}$ This technique maintains the relative simplicity of the transverse hemiarch procedure relative to total arch replacement while also providing the benefit of treating proximal DTA tear and providing DTA stabilization for improved distal aortic remodeling. This technique is not applicable, however, in cases of unresectable aortic arch or arch vessel primary tears. As the cardiac surgical community increasingly adopts DTA stent-grafting technology, it will be important to evaluate carefully the relative indications and benefits of these distal reconstructive strategies.

\section{References}

1. Zhu J-M, Qi R-D, Chen L, Liu W, Li C-N, Fan Z-M, MD, Sun L-Z. Surgery for acute type A dissection using total arch replacement combined with stented elephant trunk implantation: Preservation of autologous brachiocephalic vessels. J Thorac Cardiovasc Surg. 2015;150:101-5.

2. Di Eusanio M, Castrovinci S, Tian DH, Folesani G, Cefarelli M, Pantaleo A, et al. Antegrade stenting of the descending thoracic aorta during DeBakey type 1 acute aortic dissection repair. Eur J Cardiothorac Surg. 2014;45:967-75.

3. Kim JB, Chung CH, Moon DH, Ha GJ, Lee TY, Jung SH, et al. Total arch repair versus hemiarch repair in the management of acute DeBakey type I aortic dissection. Eur J Cardiothorac Surg. 2011;40:881-7.

4. Vallabhajosyula P, Szeto WY, Pulsipher A, Desai N, Menon R, Moeller P, et al Antegrade thoracic stent grafting during repair of acute DeBakey type I dissection promotes distal aortic remodeling and reduces late open distal reoperation rate. J Thorac Cardiovasc Surg. 2014;147:942-8.

5. Preventza O, Cervera R, Cooley DA, Bakaeen FG, Mohamed AS, Cheong BY, et al Acute type I aortic dissection: traditional versus hybrid repair with antegrade stent delivery to the descending thoracic aorta. J Thorac Cardiovasc Surg. 2014;148:119-25. 